\title{
Thermophilic campylobacter - neglected foodborne pathogens in Cambodia, laos and Vietnam
}

\begin{abstract}
Thermophilic Campylobacter are the most common bacterial cause of gastroenteritis in humans worldwide. Poultry and poultry products are the main sources for human infections. Epidemiological data concerning campylobacteriosis in Asia are limited. Overall, it is difficult to accurately assess the burden of Campylobacter infections. South-East Asia including Cambodia, Laos and Vietnam is known as a hotspot for emerging diseases. Campylobacteriosis is a problem of public health concern in these countries, hence. Epidemiological data are scarce. This is influenced by the limited number of laboratory facilities and lack of equipment and awareness in physicians and veterinarians resulting in the lack of surveys.
\end{abstract}

This review lists articles and reports on Campylobacter and campylobacteriosis in these developing third world countries. Subjects are prevalence of thermophilic Campylobacter in humans, animals and food and their resistance to several antibiotics.

Keywords: campylobacter, foodborne zoonoses, Cambodia, laos; Vietnam, antibiotic resistance
Volume 8 Issue 3 - 2017

\author{
Ngoc Huy Nguyen,' Tuan Ngoc Minh \\ Nguyen, ${ }^{2,3,4}$ Helmut Hotzel, ${ }^{2}$ Hosny El \\ Adawy, 2,5 An Quang Nguyen,' Hanh Thi \\ Tran, ${ }^{6}$ Minh Thi Hong Le, ${ }^{7}$ Herbert Tomaso, ${ }^{2}$ \\ Heinrich Neubauer, ${ }^{2}$ Hafez $\mathrm{M} \mathrm{Hafez}^{4}$ \\ 'Phutho General Hospital,Vietnam \\ ${ }^{2}$ Friedrich-Loeffler-Institut, Federal Research Institute for Animal \\ Health, Institute of Bacterial Infections and Zoonoses, Germany \\ ${ }^{3}$ Hung Vuong University, Vietnam \\ ${ }^{4}$ Institute for Poultry Diseases, Free University Berlin, Germany \\ ${ }^{5}$ Department of Poultry Disease, Faculty of Veterinary Medicine, \\ Kafrelsheikh University, Egypt \\ ${ }^{6}$ Biodiversity Conversation and Tropical Disease Research \\ Institute (BioD), Vietnam \\ ${ }^{7}$ Institute of Marine Biochemistry, Vietnam Academy of Science \\ and Technology, Vietnam
}

Correspondence: Tuan Ngoc Minh Nguyen, Friedrich-LoefflerInstitut, Federal Research Institute for Animal Health, Institute of Bacterial Infections and Zoonoses, Naumburger Str. $96 a, 07743$ Jena, Germany, Email minhtuannn@hvu.edu.vn

Received: November 28, 2017 | Published: December 20, 2017

\section{Highlights}

This review gives an overview about articles and reports on Campylobacter and campylobacteriosis in Cambodia, Laos and Vietnam. The knowledge about these objects is limited for the three countries. One topic in the literature is prevalence of thermophilic Campylobacter in humans and their relation to diarrhea. In Vietnam a prevalence rate up to $11 \%$ was reported. In Cambodia and Laos it was ever higher. Especially, children under fiveyears of age were affected. Animals and food as source for human infections play an important role. Carriage of Campylobacter by different animal species and contamination rate of meat are generally high and can reach more than $70 \%$. Resistance to antibiotics is of public health concern. High rates of resistance to nalidixic acid, erythromycin, tetracycline and ciprofloxacin were detected reaching sometimes $100 \%$ of isolates.

\section{Introduction}

Zoonoses are diseases and infections which can be transmitted from animals to humans or vice versa. Over 200 pathogens are recognized as zoonotic agents and classified as foodborne and nonfoodborne agents. Zoonotic foodborne pathogens may cause human diseases after uptake of contaminated food or water. Several of these microorganisms can be found in the intestinal tract of healthy foodproducing animals e. g. thermophilic Campylobacter species.

Thermophilic Campylobacter species are the most common bacterial cause of gastroenteritis in humans worldwide. Incidence and prevalence of campylobacteriosis have increased in both, developed and developing countries, over the last 10years. ${ }^{1}$ A dramatic increase in the number of reported cases was recognized in Australia, Europe, and North America. In the United States, an incidence of 14.3 campylobacteriosis cases per 100.000 inhabitants was reported for the period between 1996 and 2012. ${ }^{2}$ In Quebec, Canada an annual incidence of 35.2 cases per 100,000persons was reported. ${ }^{3}$ In Europe, Campylobacter has become the most frequently reported bacterial pathogen causing gastrointestinal infections in humans since 2005. In 2013, 214,779 confirmed cases were reported by the member states of the European Union (EU) which correlated with a notification rate of 64.8 per 100,000 inhabitants. ${ }^{4}$ Hence, the number of fatal cases was very low with $0.05 \%$. Data from African countries are limited and indicate that Campylobacter infections are most prevalent in children. In a study in Malawi, $14 \%$ of non-diarrheic children and $28 \%$ children with diarrhea were PCR positive for $C$. jejuni and $C$. coli. ${ }^{5}$ C. jejuni and C. coli were also found to be endemic in children in Madagascar and Kenya ${ }^{6,7}$. Epidemiological data concerning campylobacteriosis in Asia are limited. A study from China reported that $5 \%$ of diarrheic patients were PCR positive for C. jejuni. The highest prevalence was detected in the cohort of children younger than 7years. ${ }^{8}$ Also in Japan and India Campylobacter infections occur quite frequently. ${ }^{9,10}$ Overall, it is difficult to accurately assess the burden of Campylobacter infections in Asia owing to insufficient epidemiological data.

International travel, consumption of undercooked chicken and products thereof, environmental exposure, and direct contact with farm animals were recognized as risk factors for human campylobacteriosis. ${ }^{11}$ The most important sources of foodborne 
campylobacteriosis are consumption of contaminated food, especially poultry products, unpasteurized milk and water. Broilers are the main source for thermophilic campylobacters to humans. ${ }^{12}$ Studies in Switzerland estimated that $71 \%$ of human cases were caused by uptake of contaminated chicken meat. ${ }^{13,14}$ The UK Food Standards Agency found $72.9 \%$ of fresh retail chicken Campylobacter positive with nearly $20 \%$ being highly contaminated..$^{15}$ Besides broilers, turkeys and ducks, and also cattle and pigs serve as reservoirs of thermophilic Campylobacter. Campylobacter contaminated water was responsible for outbreaks of human campylobacteriosis, but also for transmission within animal populations. ${ }^{1}$

South-East Asia is known as a hotspot for emerging diseases. Part of the region is former French Indochina including Cambodia (Kingdom of Cambodia), Laos (Lao People's Democratic Republic) and Vietnam (Socialist Republic of Vietnam) with a shared history since the 19th century. All three countries are developing third world countries suffering the aftermath of the Vietnam War. Campylobacteriosis is a problem of public health concern in these countries, hence. Epidemiological data are scarce. This may be caused by the limited number of laboratory facilities and lack of equipment and awareness in physicians and veterinarians resulting in the lack of surveys. A recent review of foodborne bacterial and parasitic zoonosis in Vietnam summarized a number of studies on thermophilic Campylobacter infections ${ }^{16}$ but no data on the prevalence of campylobacteriosis are available for Laos and Cambodia.

Therefore, in this review we summarize literature on Campylobacter affecting human and animal populations, their prevalence as foodborne pathogen and the resistance to antibiotics in these countries from 1971 to 2016. We delineate knowledge and capability gaps, which will foster new research and surveillance programs. This will help to tackle the impact on public health that is caused by Campylobacter infections in the respective countries.

\section{Methods and research data}

Information presented in this review was collected by searching published studies on database including CABDIRECT, Science Direct, Pubmed and Google with keywords "Campylobacter and Vietnam", "Campylobacter and Laos", "Campylobacter and Cambodia". The searched publications were reviewed and relevant information was retrieved. All Articles or studies provided information on prevalence of thermophilic Campylobacter in humans, animals or food and/or information on antibiotic resistance in these bacteria. All articles and studies were in relation to South-East Asian countries of Cambodia, Laos and/or Vietnam.

\section{Results and discussion}

Articles on campylobacter in cambodia, laos and vietnam

Thirty one publications were retrievable in Pubmed and other database concerning Campylobacter in Cambodia, Laos and Vietnam between 1971 and 2016. Nineteen articles were related to Vietnam, 3 articles to Laos and 9 articles to Cambodia. Eleven articles were related to antibiotic resistance but most of the papers dealt with investigations of the prevalence of Campylobacter in humans, animals and food.

\section{Campylobacterin humans}

An overview about papers concerning Campylobacter in humans in Vietnam, Laos and Cambodia is given in Table 1. The main source of human campylobacteriosis is the consumption of chicken meat, but meat of other species can also be contaminated with Campylobacter. Identification of thermophilic campylobacters was carried out by cultivation in combination with biochemical methods. Only recently, identification and differentiation by PCR assays was introduced. Detection rates ranged between 0 and $12 \%$ depending on country and the method of detection. C. jejuni was detected more often than $C$. coli. Children under 5years of age are most frequently affected. The risk of infections seems to be correlated with undernutrition, poor hygiene, keeping of animals in the house, manure and wet litter in house yards and contaminated drinking water. ${ }^{17}$ In Cambodia, 12\% of 681 human faecal samples were tested positive by PCR assays. ${ }^{18}$ Rates for Vietnam and Laos were below $5 \%$. In an investigation in children with and without diarrhea in Ho Chi Minh City, Vietnam, $2.2 \%$ of stool samples were positive, but in the control group without diarrhea $2.6 \%$ samples were also found positive. ${ }^{19} \mathrm{~A}$ similar result was reported from Cambodia. In Phnom Penh $4.7 \%$ C. jejuni and $1.5 \%$ C. coli were detected in diseased children at an age under 5 years and $6.2 \%$ C. jejuni and $2.4 \%$ C. coli in the control group. ${ }^{20}$ It seems that Campylobacter is widespread in the population, but the mere presence of the bacteria in the gut is not inevitable related to clinical symptoms of campylobacteriosis.

In a detailed study from Cambodia, Osbjer et al., ${ }^{18}$ could not detect Campylobacter in 681 stool samples by cultivation of frozen samples. ${ }^{18}$ Hence, 66 C. jejuni and 16 C. coli were identified by multiplex PCR. In the group of children up to 15 years, Campylobacter was detected in $18.8 \%$ of the samples whereas only $7 \%$ to $8 \%$ of those of male and female group over 15 years were $C$. positive. Risk factors for human campylobacteriosis were slaughtering of domestic animals, allowance of animal access to sleeping and food preparation areas and eating of undercooked meat. ${ }^{17}$

\section{Campylobacterin animals and meat}

In an investigation from the Mekong delta, Vietnam, the prevalence of Campylobacter in faeces of chickens, ducks and pigs was reported to be $31.9 \%, 23.9 \%$, and $53.7 \%$, respectively. ${ }^{21}$ Similar results were found in Cambodia. ${ }^{18}$ In $41.3 \%$ of swab and faeces samples of chickens, ducks, pigs and cattle Campylobacter was detected by multiplex PCR. $56.1 \%$ of chicken and $23.8 \%$ of duck samples were positive. $72.2 \%$ of pigs but only $5.3 \%$ of cattle samples were tested positive for $C$. jejuni and $C$. coli, respectively. $C$. jejuni was the dominant species in chickens and ducks, $C$. coli was more prevalent in pigs. The low prevalence rate of Campylobacter in cattle (5.3\%) was similar to that in buffaloes in Laos i. e. $2 \% .{ }^{22} \mathrm{~A}$ remarkable difference was observed between cultivation and PCR assays. In contrast to 352 samples that were assessed to be positive by PCR assays (41.3\%) only 106 samples were identified as Campylobacter positive by cultivation $(12.4 \%)$. Cultivation of Campylobacter is difficult at least under field conditions because of their sensitivity to oxygen and changes in temperature.

Contamination rates of poultry products with thermophilic Campylobacter were determined to be between $15 \%$ and $35 \%$ in Vietnam (Table 2). Schwan, 2010 found $76.0 \%$ of swabs of chickens positive for Campylobacter, but none of the investigated meat samples was contaminated. ${ }^{23}$ In Phnom Penh, Cambodia, was shown that $80.9 \%$ of poultry carcasses were contaminated. ${ }^{24}$ The result was obtained by cultivation of Campylobacter. A lower contamination rate of $35.0 \%$ was reported for poultry products from markets in the capital of Cambodia. ${ }^{25}$ C. jejuni (44.4\%), C. coli (36.5\%), C. lari $(15.9 \%)$ and C. upsaliensis $(3.2 \%)$ were identified among 63 Campylobacter isolates. 
Table I Studies concerning presence of thermophilic Campylobacter in humans

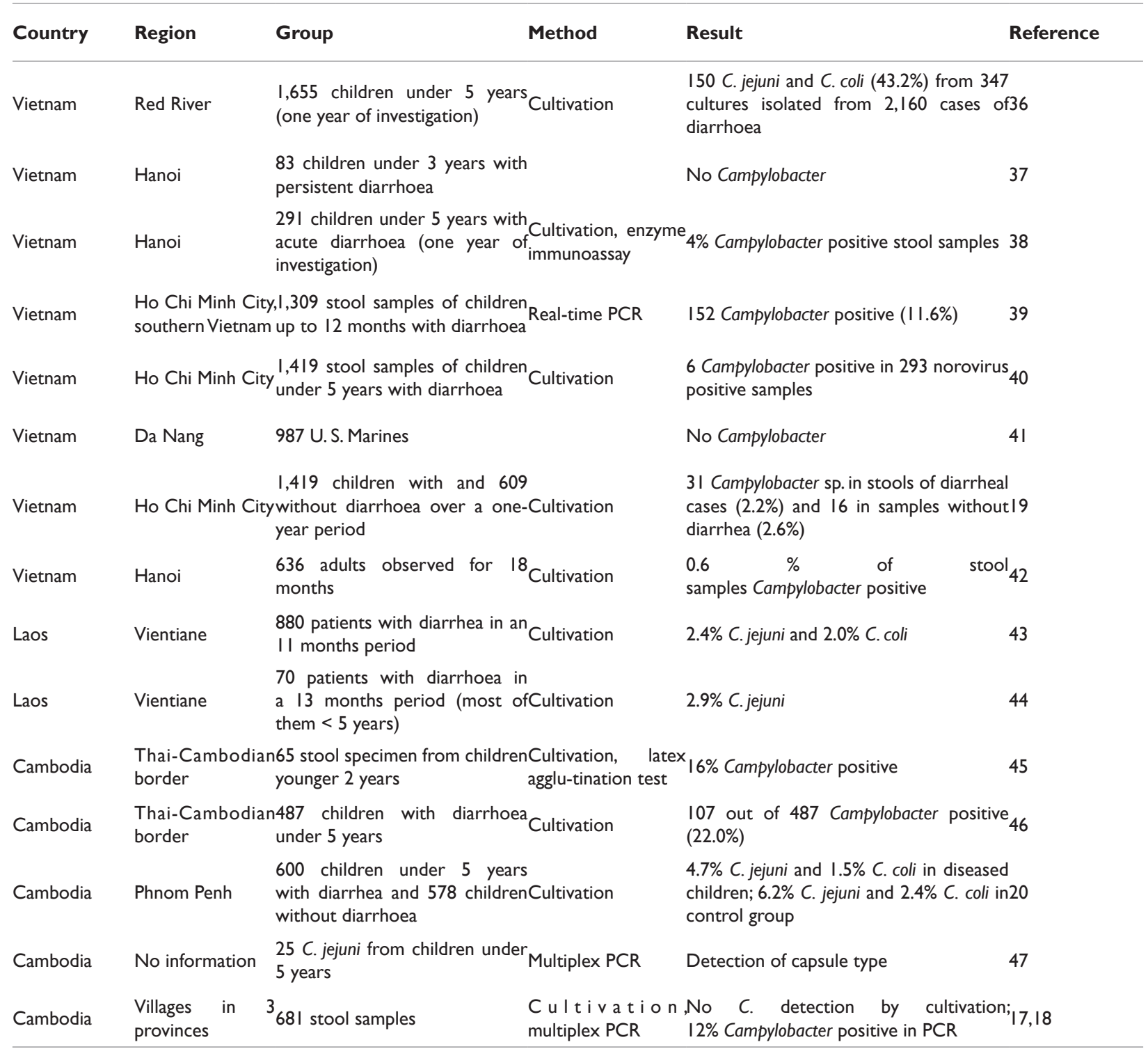

Table 2 Studies concerning antibiotic resistance of thermophilic Campylobacter of different origin

\begin{tabular}{|c|c|c|c|c|c|}
\hline Country & Region & Source & $\begin{array}{l}\text { Number of Isolates } \\
\text { Method of Investigation }\end{array}$ & Resistance Rate to & Reference \\
\hline Vietnam & - & Human & $\begin{array}{l}88 \text { isolates; } \\
\text { MIC agar-plate dilution test }\end{array}$ & $\begin{array}{l}\text { NA: } 7 \% \\
\text { CIP: } 7 \% \\
\text { AZM: } 0 \%\end{array}$ & 27 \\
\hline Vietnam & Hanoi & Human & & CIP: $27 \%$ & 38 \\
\hline Vietnam & Mekong delta & Chicken & $\begin{array}{l}22 \text { C. jejuni and } 6 \text { C. coli; } \\
\text { Broth microdilution test }\end{array}$ & $\begin{array}{l}\text { NA: } 64 \% \text { b); } 100 \% \text { c) } \\
\text { ERY: } 0 \% \text { b); } 33 \% \text { c) } \\
\text { CIP: } 64 \% \text { b); } 100 \% \text { c) } \\
\text { GEN: } 9 \% \text { b); 33\% c) } \\
\text { STR: }(4 \% \text { b); } 50 \% \text { c) } \\
\text { TET: } 68 \% \text { b); } 83 \% \text { c) }\end{array}$ & 23 \\
\hline
\end{tabular}


Table continued..

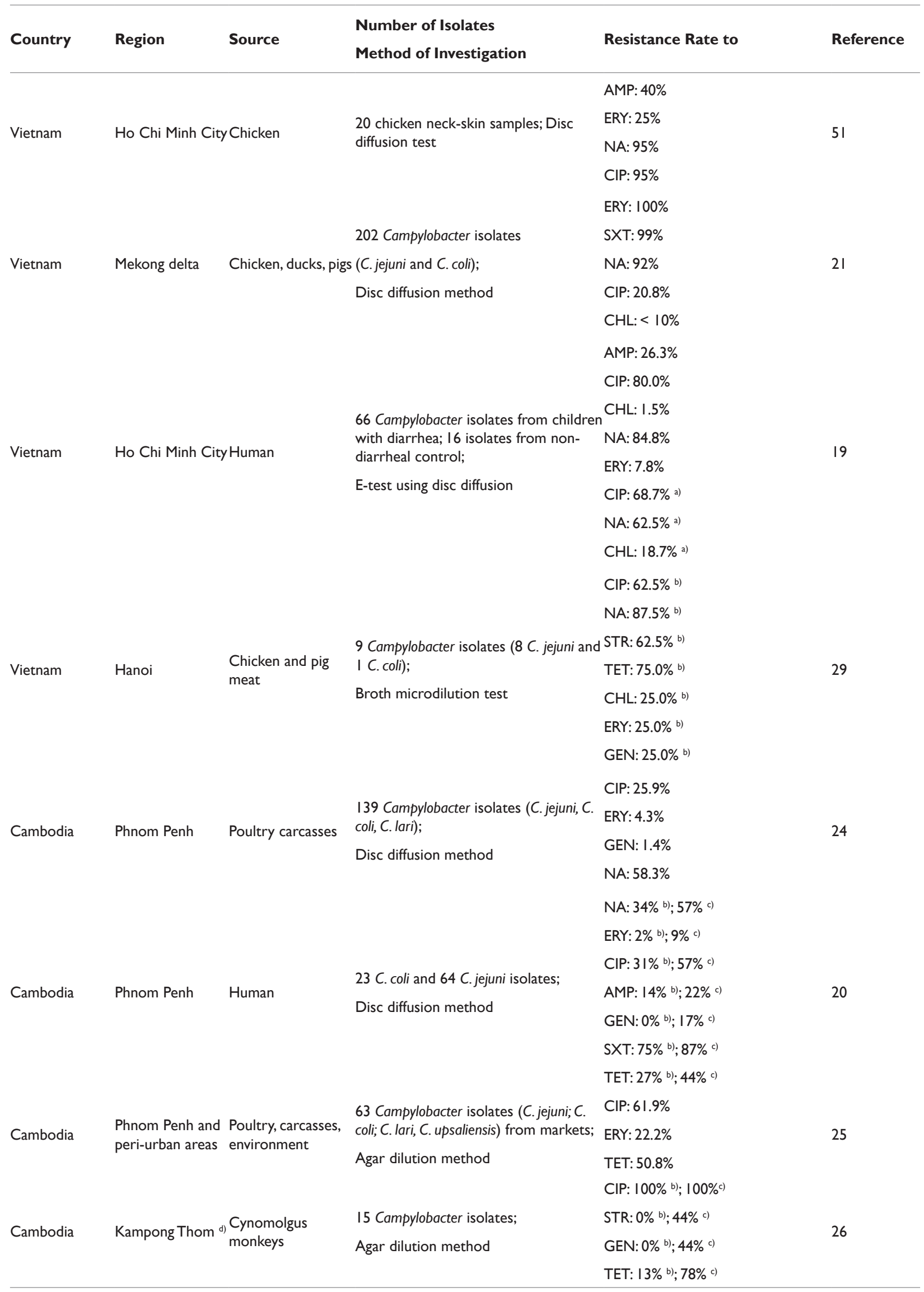


NA: Nalidixic Acid; CIP: Ciprofloxacin; AZM: Azithromycin; AMP: Ampicillin; ERY: Erythromycin; SXT: SulfamethoxazoleTrimethoprim; CHL: Chloramphenicol; STR: Streptomycin; TET: Tetracycline; GEN: Gentamicin

Resistance rates of Cambodian campylobacters from chicken to ciprofloxacin reached $90.0 \%$ for $C$. lari isolates and was lower for $C$. jejuni and C. coli with $60.7 \%$ and $52.2 \%$, respectively. ${ }^{25} C$. coli $(30.4 \%)$ showed a higher resistance rate to erythromycin in comparison to $C$. jejuni $(17.9 \%)$. Resistance to tetracycline varied around $50 \%$ whereupon $C$. coli showed the highest value (56.5\%). Campylobacter isolated from faeces of monkeys were $100 \%$ sensitive to erythromycin and chloramphenicol. ${ }^{26}$ Archawakulathep et al., ${ }^{30} 2014$ gave a good overview of perspectives on antimicrobial resistance in livestock and livestock products in ASEAN countries. ${ }^{30}$

Consequence of finding of fluoroquinolone in imported basa catfish from Vietnam was the stop of sale of 350 tons of seafood in the US by the U. S. Food and Drug Administration (FDA) in $2005 .{ }^{31}$ Motivation was the emerging of resistance to enrofloxacin in Campylobacter causedby treatment of chickens and turkeys with this antimicrobial agent in poultry production and the risk for human health.

\section{Other topics}

Recently, five genomes of Campylobacter jejuni isolates from Vietnam were sequenced. Some of these isolates had a cluster of genes of the type-6 secretion system (T6SS) which play roles in pathogenpathogen and host-pathogen interactions. T6SS is associated with virulence, cell adhesion and cytotoxicity toward erythrocytes. Using the marker gene hcp (haemolysin co-regulated protein) the T6SS was detected in more than $70 \%$ of Vietnamese human and chicken isolates. ${ }^{32}$

Another study gave a detailed characterization of Vietnamese Campylobacter isolates. ${ }^{29}$ Investigations concerning genotyping and antimicrobial resistance of Campylobacter isolates were carried out using fla A typing, MLST and DNA microarray assays. Resistance of Campylobacter to several antibiotics was determined phenotypically and by molecular biological methods. A limitation was the low number of isolates. In a study concerning the regional risks and seasonality in travel-associated campylobacteriosis in East Asia including Cambodia, Laos and Vietnam the risk was estimated to be 386 infections per 100,000 Swedish travelers per year. This is the highest value in the world apart from the Indian subcontinent with 1,253 cases per 100,000 travelers per year. ${ }^{33}$

In an evaluation study of gastrointestinal pathogens in stool samples from diarrheic patients the usefulness of a multipanel pathogen identification system was shown. It represented a sensitive, specific and easy approach as an alternative to classical detection methods. ${ }^{34}$

\section{Conclusion}

Little information about Campylobacter was reported in the past in the three South East Asian countries of Cambodia, Laos and Vietnam. Often, investigations were related to human infections especially in children of young age in big cities like Hanoi, Vientiane, Phnom Penh or Ho Chi Minh City. Knowledge about the prevalence of Campylobacter in humans and poultry and the antibiotic resistance is much better in Vietnam than in Laos or Cambodia. However, there exist no data about the prevalence of Campylobacter in milk or water sources, although Campylobacter contamination in both could be a risk for humans. Moreover, the common habit of consumption of unpasteurized milk in children under 5year of age underlines the relevance of this potential route of transmission in these countries. However, food safety awareness and concepts are existing. ${ }^{35}$ Surveillance and collaborative research within the South East Asian countries can clarify the epidemiology of foodborne infections like campylobacteriosis in humans. It can be also important for control of bacterial contamination in livestock and food of animal origin.

Prerequisite of improvement of food safety and as a consequence of human health is the introduction of modern diagnostics. PCR assays are rapid, reliable and comparably cheap, but especially in Laos molecular techniques are practically not in use yet and there is a substantial lack of laboratory infrastructure and equipment in all three countries. An increasing problem is antibiotic resistance in bacteria like Campylobacter. In the EU, antibiotic use of antibiotics as growth promotors in food animals was completely banned several years ago. National monitoring and control programmes for antimicrobial resistance in foodborne pathogens have not been established in ASEAN countries yet..$^{30,36-51}$ Limited data on the amount of antibiotics used in the farming industry exist, because there is no effective control, policy or regulation. In summary, national surveillance programs and international collaborations are needed to address the substantial gaps in knowledge about the epidemiology of campylobacteriosis in developing countries such as Cambodia, Laos and Vietnam. Establishment of at least one National Reference Laboratory with modern equipment and well trained personnel in each country is recommended.

\section{Acknowledgements}

This work was supported by Friedrich-Loeffler-Institut, Institute of Bacterial Infections and Zoonoses, Germany and Vietnam Ministry of Agriculture and Rural Development.

\section{Conflicts of interest}

No competing financial interests exist.

\section{Funding}

None.

\section{References}

1. Kaakoush NO, Castano-Rodriruez N, Mitchell HM, et al. Global epidemiology of Campylobacter infection. Clin Microbiol Rev. 2015;28(3):687-719.

2. Gilliss $\mathrm{D}$, Cronquist $\mathrm{AB}$, Cartter $\mathrm{M}$, et al. Incidence and trends of infection with pathogens transmitted commonly through food-foodborne diseases active surveillance network, 10. U. S. sites, 1996-2012. MMWR Rep. 2013;62: 283-287.

3. Arsenault J, Berke O, Michel P, et al. Environmental and demographic risk factors for campylobacteriosis: do various geographical scales tell the same story? BMC Infect Dis. 2012;12:318.

4. EFSA/ECDC. The European Union summary report on trends and sources of zoonoses, zoonotic agents and food-borne outbreaks in 2013. EFSA J. 2015;13:3991.

5. Mason J, Iturriza-Gomara M, O’Brien SJ, et al. Campylobacter infection in children in Malawi is common and is frequently associated with enteric virus co-infections. PLoS One. 2013;8(3):e59663.

6. Randremanana RV, Randrianirina F, Sabatier P, et al. Campylobacter infection in a cohort of rural children in Moramanga, Madagascar. BMC Infect Dis. 2014;14:372. 
7. Swierczewski BE, Odundo EA, Koech MC, et al. Enteric pathogen surveillance in a case-control study of acute diarrhea in Kisii Town, Kenya. J Microbiol Med. 2013;62(pt 11):1774-1776.

8. Huang JL, Xu HY, Bao GY, et al. Epidemiological surveillance of Campylobacter jejuni in chicken, dairy cattle and diarrhea patients. Epidemiol Infect. 2009;137(8):1111-1120.

9. Kubota K, Kasuga F, Iwasaki E, et al. Estimating the burden of acute gastroenteritis and foodborne illness caused by Campylobacter, Salmonella, and Vibrio parahaemolyticus by using population-based telephone survey data, Miyagi Prefecture, Japan, 2005 to 2006. J Food Prot. 2011;74(10):1592-1598.

10. Mukherjee P, Ramamurthy T, Bhattacharya MK, et al. Campylobacter jejuni in hospitalized patients with diarrhea, Kolkata, India. Emerg Infect Dis. 2013;19(7):1155-1156.

11. Domingues AR, Pires SM, Halasa T, et al. Source attribution of human campylobacteriosis using a meta-analysis of case-control studies of sporadic infections. Epidemiol Infect. 2012;140(6):970-981.

12. Skirrow MB. Campylobacter enteritis: a "new" disease. $\mathrm{Br}$ Med J. 1977;2(6078):9-11.

13. Kittl S, Heckel G, Korczak BM, et al. Source attribution of human Campylobacter isolates by MLST and fla-typing and association of genotypes with quinolone resistance. PLoS One. 2013;8(11):e81796.

14. Wei W, Schüpbach G, Held L. Time-series analysis of Campylobacter incidence in Switzerland. Epidemiol Infect. 2015;143(9):1982-1989.

15. Food Standards Agency (FSA). A microbiological survey of Campylobacter contamination in fresh whole UK produced chilled chickens at retail sale - an interim report to cover Quarters 1\&2. Food Standards Agency, London, UK. 2014.

16. Carrique-Mas JJ, Bryant JE. A review of foodborne bacterial and parasitic zoonoses in Vietnam. EcoHealth. 2013;10(4):465-489.

17. Osbjer K, Boqvist S, Sokerya S, et al. Risk factors associated with Campylobacter detected by PCR in humans and animals in rural Cambodia. Epidemiol Infect. 2016;144(14):1-10.

18. Osbjer K, Tano E, Chhayheng L, et al. Detection of Campylobacter in human and animal field samples in Cambodia. APMIS. 2016;124(6):508515 .

19. Thompson CN, Phan MVT, Hoang NVM, et al. A prospective multicenter observational study of children hospitalized with diarrhea in Ho Chi Minh City, Vietnam. Am J Trop Med Hyg. 2015;92(5):1045-1052.

20. Meng CY, Smith BL, Bodhidatta L, et al. Etiology of diarrhea in young children and patterns of antibiotic resistance in Cambodia. Pediatr Infect Dis J. 2011;30:331-335.

21. Carrique-Mas JJ, Bryant JE, Cuong NV, et al. An epidemiological investigation of Campylobacter in pig and poultry farms in the Mekong delta of Vietnam. Epidemiol Infect. 2014;142(7):1425-1436.

22. Boonmar S, Chanda C, Markvichitr K, et al. Prevalence of Campylobacter spp. in slaughtered cattle and buffaloes in Vientiane, Lao People's Democratic Republic. J Vet Med Sci. 2007;69(8):853-855.

23. Schwan P. Prevalence and antibiotic resistance of Campylobacter spp. in poultry and raw meat in the Can Tho Province, Vietnam. SLU Examensarbete. 2010.

24. Lay KS, Vuthy Y, Song P, et al. Prevalence, numbers and antimicrobial susceptibilities of Salmonella serovars and Campylobacter spp. in retail poultry in Phnom Penh, Cambodia. J Vet Med Sci. 2011;73(3):325-329.

25. Otto P. CambodiaCase Study: An integrated surveillance study of AMR in Salmonella subspp, Campylobacter spp, Escherichia coli and Enterococcus spp in poultry. Regional Workshop on the Use of Antimicrobials in Animal Production and AMR in the Asia-Pacific Region. Negombo, Sri Lanka. 2012; pp.22-23.
26. Koga T, Aoki W, Mizuno $\mathrm{T}$, et al. Antimicrobial resistance in Campylobacter coli and Campylobacter jejuni in cynomolgus monkeys (Macaca fascicularis) and eradication regimens. J Microbiol Immunol Infect. 2015;50(1):75-82.

27. Isenbarger DW, Hoge CW, Srijan A, et al. Comparative antibiotic resistance of diarrheal pathogens from Vietnam and Thailand, 19961999. Emerg Infect Dis. 2012;8(2):175-180.

28. European Food Safety Authority (EFSA). Scientific opinion on chloramphenicol in food and feed. EFSA J. 2014;12:3907.

29. Nguyen TNM, Hotzel H, El-Adawy H, et al. Genotyping and antibiotic resistance of thermophilic Campylobacter isolated from chicken and pig meat in Vietnam. Gut Pathog. 2016;8:19.

30. Archawakulathep A, Kim CTT, Meunsene D, et al. Perspectives on antimicrobial resistance in livestock and livestock products in ASEAN countries. Thai J Vet Med. 2014;44:5-13.

31. Lousiana recalls and stops sale of some 700.000 pounds of fish from Vietnam pending investigation into use of antibiotic banned by the FDA in July. Campylobacter Blog - Surveillance \& Analysis on Campylobacter News \& Outbreaks. 2005.

32. Harrison JW, Dung TTN, Siddiqui F, et al. Identification of possible virulence marker from Campylobacter jejuni isolates. Emerg Infect Dis. 2014;20(6):1026-1029.

33. Ekdahl K, Andersson Y. Regional risks and seasonality in travelassociated campylobacteriosis. BMC Infect Dis. 2004;4(1):54.

34. Duong VT, Phat VV, Tuyen HT, et al. Evaluation of Luminex xTAG Gastrointestinal Pathogen Panel Assay for detection of multiple diarrheal pathogens in fecal samples in Vietnam. $J$ Clin Microbiol. 2016;54(4):1094-1100.

35. Padungtod P, Kadohira M, Hill G. Food safety concepts and foodborne bacteria in food animals in Thailand, Lao PDR and Vietnam. Chiang Mai Vet J. 2007;5:113-122.

36. Isenbarger DW, Hien BT, Ha HT, et al. Prospective study of the incidence of diarrhoea and prevalence of bacterial pathogens in a cohort of Vietnamese children along the Red River. Epidemiol Infect. 2001;127(2):229-236.

37. Ngan PK, Khanh NG, Tuong CV, et al. Persistent diarrhea in Vietnamese children: a preliminary report. Acta Paediatr 81 Suppl. 1992;381:124 126.

38. Bodhidatta L, Lan NT, Hien BT, et al. Rotavirus disease in young children from Hanoi, Vietnam. Pediatr Infect Dis J. 2007;26(4):325328.

39. Anders KL, Thompson CN, Thuy NTV, et al. The epidemiology and aetiology of diarrhoeal disease in infancy in southern Vietnam: a birth cohort study. Int J Infect Dis. 2015;35:3-10.

40. My PVT, Thompson C, Phuc HL, et al. Endemic norovirus infections in children, Ho Chi Minh City, Vietnam, 2009-2010. Emerg Infect Dis. 2013;19(6):977-980.

41. Forman DW, Tong MJ, Murrell KD, et al. Etiologic study of diarrheal disease in Vietnam. Am J Trop Med Hyg. 1971;20(4):598-601.

42. Trang DT, Hien BTT, Molbak K, et al. Epidemiology and aetiology of diarrhoeal diseases in adults engaged in wastewater-fed agriculture and aquaculture in Hanoi, Vietnam. Trop Med Int Health 12 Suppl. 2007;2:23-33.

43. Yamashiro T, Nakasone N, Higa N, et al. Etiological study of diarrheal patients in Vientiane, Lao Peoples's Democratic Republic. J Clin Microbiol. 1998;36(8):2195-2199.

44. Phetsouvanh R, Midorikawa Y, Nakamura S. The seasonal variation in the microbial agents implicated on the etiology of diarrheal diseases among children in Lao People's Democratic Republic. Southeast Asian J Trop Med Public Health. 1990;30(2):319-323. 
45. Nordlander E, Phuphaisan S, Bodhidatta L, Arthur J, Echeverria P (1990) Microscopic examination of stools and a latex slide agglutination test for the rapid identification of bacterial enteric infections in Khmer children. Diag Microbiol Infect Dis 13(3): 273-276.

46. Arthur JD, Bodhidatta L, Echeverria P, et al. Diarrheal disease in Cambodian children at a camp in Thailand. Am J Epidem. 1992;135(5):541-551.

47. Poly F, Serichantalergs O, Kuroiwa J, et al. Updated Campylobacter jejuni capsule PCR multiplex typing system and its application to clinical isolates from South and Southeast Asia. PLoS One. 2015;10:e144349.

48. Dao HTA, Yen PT. Study of Salmonella, Campylobacter, and Escherichia coli contamination in raw food available in factories, schools, and hospital canteens in Hanoi, Vietnam. Ann NY Acad Sci. 2006;1081:262265.
49. Huong LQ, Hanh TT, Cam PD, et al. Study on the prevalence of Campylobacter spp. from chicken meat in Hanoi, Vietnam. Ann NY Acad Sci. 2006;1081:273-275.

50. Bao VN, Fries R, Zessin KH, et al. Salmonella and Campylobacter in broiler carcasses in Vietnam. Proc 11th Int Symp Vet Epidemiol Econom. 2006.

51. Garin B, Gouali M, Wouafo M, et al. Prevalence, quantification and antimicrobial resistance of Campylobacter spp. on chicken neck-skins at points of slaughter in 5 major cities located on 4 continents. Int J Food Microbiol. 2012;157(1):102-107. 